\title{
Pleuroparenchymal fibroelastosis in systemic sclerosis: prevalence and prognostic impact
}

\author{
Martina Bonifazi ${ }^{1,2,3}$, Nicola Sverzellati ${ }^{4}$, Eva Negri ${ }^{5}$, Joseph Jacob (10), \\ Ryoko Egashira ${ }^{8}$, Joanna Moser ${ }^{9}$, Sara Piciucchi $\mathbb{1}^{10}{ }^{10}$. Federico Mei ${ }^{2}$, \\ Angelo De Lauretis ${ }^{3,11}$, Dina Visca ${ }^{3,12}$, Nicole Goh ${ }^{13,14}$, Matteo Bonini ${ }^{15,16}$, \\ Laura Cirilli ${ }^{1}$, Carlo La Vecchia ${ }^{17}$, Felix Chua ${ }^{3}$, Vasileios Kouranos ${ }^{3}$, \\ George Margaritopoulos ${ }^{3,18}$, Maria Kokosi ${ }^{3}$, Toby M. Maher ${ }^{3}$, \\ Stefano Gasparini ${ }^{1,2}$, Armando Gabrielli ${ }^{19}$, Athol U. Wells ${ }^{3}$ and \\ Elisabetta A. Renzoni ${ }^{3}$
}

@ERSpublications

We provide a thorough assessment of pleuroparenchymal fibroelastosis (PPFE) prevalence, severity and clinical impact in two large cohorts of scleroderma patients (total $n=359$ ). PPFE was present in $18 \%$ of patients and independently predicted mortality. https://bit.ly/2xDVvG3

Cite this article as: Bonifazi M, Sverzellati N, Negri E, et al. Pleuroparenchymal fibroelastosis in systemic sclerosis: prevalence and prognostic impact. Eur Respir J 2020; 56: 1902135 [https://doi.org/10.1183/ 13993003.02135-2019].

ABSTRACT Interstitial lung disease (ILD) in systemic sclerosis (SSc) is a major cause of morbidity and mortality, mostly presenting as non-specific interstitial pneumonia. Little is known about the prevalence of pleuroparenchymal fibroelastosis (PPFE), a specific entity affecting the visceral pleura and subpleural parenchyma. We set out to estimate PPFE prevalence in two large cohorts of SSc patients and to assess its impact on survival and functional decline.

A total of 359 SSc patients, derived from two referral centres in two different countries (UK and Italy), were included. The first available high-resolution computed tomography scan was independently evaluated by two radiologists blind to clinical information, to quantify ILD extent, freestanding bronchial abnormalities, and lobar percentage involvement of PPFE on a four-point categorical scale. Discordant scores were adjudicated by a third scorer. PPFE extent was further classified as limited $(\leqslant 2 / 18)$ or extensive $(>2 / 18)$. Results were evaluated against functional decline and mortality.

The overall prevalence of PPFE in the combined SSc population was $18 \%$ (11\% with extensive PPFE), with no substantial difference between the two cohorts. PPFE was significantly linked to free-standing bronchial abnormalities ( $61 \%$ versus $25 \%$ in PPFE versus no PPFE; $\mathrm{p}<0.0001$ ) and to worse survival, independently of ILD severity or short-term lung function changes (HR 1.89, 95\% CI 1.10-3.25; p=0.005).

In the current study, we provide an exhaustive description of PPFE prevalence and clinical impact in the largest cohort of SSc subjects published so far. PPFE presence should be carefully considered, due to its significant prognostic implications. 


\section{Introduction}

Systemic sclerosis (SSc, Scleroderma) is a rare, immune-mediated disorder, characterised by microvascular injury, circulating autoantibodies and fibroblast activation, leading to fibrosis of the skin and visceral organs [1]. Lung involvement, including interstitial lung disease (ILD) and/or pulmonary hypertension, is the leading cause of morbidity and mortality [2]. ILD may range from subclinical to severe progressive lung fibrosis. Baseline functional impairment and short-term pulmonary function test (PFT) trends, along with ILD extent on high-resolution computed tomography (HRCT), are currently the most informative prognostic tools in routine clinical practice [3,4]. Morphologically, nonspecific interstitial pneumonia (NSIP) is the most common pattern, while a usual interstitial pneumonia (UIP) pattern is seen in a minority. Little is known about prevalence and prognostic value of other parenchymal abnormalities [5]; in particular, no large-scale study has assessed presence and potential impact of pleuroparenchymal fibroelastosis (PPFE) in SSc.

PPFE is a specific clinical-pathological entity affecting the visceral pleura and the subpleural parenchyma with an upper-lobe predilection, characterised by elastin-rich intra-alveolar fibrosis and scattered fibroblastic foci [6]. Its pathogenesis is unclear, but the heterogeneous spectrum of clinical presentation and behavior suggests that it may represent the final expression of a variable interplay between immune dysregulation, environmental exposure and genetic predisposition [6]. PPFE can present as an idiopathic form, included in the latest American Thoracic Society (ATS)/European Respiratory Society (ERS) classification of idiopathic interstitial pneumonias [7], or in association with a variety of different conditions, including infection, lung and bone marrow transplantation and autoimmune diseases $[8,9]$. Moreover, PPFE features are observed in association with other interstitial lung diseases, including idiopathic pulmonary fibrosis (IPF) [10, 11], hypersensitivity pneumonitis [12] and familial forms of pulmonary fibrosis [13].

The aims of the present study were to estimate the prevalence of PPFE in large unselected consecutive cohorts of SSc patients, to assess its potential impact on survival and functional decline, and to evaluate the correlation between PPFE and selected radiological/clinical features.

\section{Materials and methods}

Two cohorts were included: one from the Royal Brompton Hospital Interstitial Lung Diseases Unit, London, UK (RBH cohort), the other from the rheumatological centre of the Clinica Medica of University Hospital "Ospedali Riuniti”, Ancona, Italy (Ancona cohort). Ethical approvals for this study of clinically indicated HRCT and pulmonary function data were obtained from the Institutional Ethics Committees of $\mathrm{RBH}$ and of "Ospedali Riuniti", respectively.

\section{Patients and clinical information}

Consecutive patients with a diagnosis of SSc according to recommended criteria [14], presenting to RBH between 1990 and 2011 and to Ancona between 2002 and 2014 were eligible for inclusion in the absence of the following exclusion criteria: 1) overlap with other connective tissue disease; 2) unavailability of the HRCT scan; and 3) predominant radiological features suggestive of vasculopathy (supplementary material).

Demographic factors and selected disease-related characteristics were collected for all SSc cases. These included: age, sex, smoking history, disease duration (defined as months from the onset of the first

Affiliations: ${ }^{1}$ Dept of Biomedical Sciences and Public Health, Università Politecnica delle Marche, Ancona, Italy. ${ }^{2}$ Pulmonary Disease Unit, Dept of Internal Medicine, Azienda Ospedali Riuniti, Ancona, Italy. ${ }^{3}$ Interstitial Lung Disease Unit, Royal Brompton Hospital, Imperial College, London, UK. "Radiology, Dept of Medicine and Surgery, Università di Parma, Parma, Italy. "Dept of Biomedical and Clinical Sciences "Luigi Sacco", Università degli Studi di Milano, Milan, Italy. ${ }^{6}$ Dept of Respiratory Medicine, University College London, London, UK. ${ }^{7}$ Centre for Medical Image Computing, University College London, London, UK. ${ }^{8}$ Dept of Radiology, Faculty of Medicine, Saga University, Saga city, Japan. ${ }^{9}$ Dept of Radiology, St George's University Hospitals NHS Foundation Trust, London, UK. ${ }^{10}$ Radiology Unit, Ospedale GB Morgagni, Forli, Italy. ${ }^{11}$ Pulmonary Diseases Unit, Azienda Ospedaliera "Guido Salvini", Garbagnate Milanese, Italy. ${ }^{12}$ Division of Pulmonary Rehabilitation, Istituti Clinic Scientifici Maugeri, IRCCS, Tradate, Italy. ${ }^{13}$ Dept of Respiratory Medicine, Austin Hospital, Melbourne, Australia. ${ }^{14}$ Institute for Breathing and Sleep, Melbourne, Australia. ${ }^{15}$ National Heart and Lung Institute (NHLI), Imperial College London and Royal Brompton Hospital, London, UK. ${ }^{16}$ Dept of Cardiovascular and Thoracic Sciences, Fondazione Policlinico Universitario A.Gemelli-IRCCS, Università Cattolica del Sacro Cuore, Rome, Italy. ${ }^{17}$ Dept of Clinical Sciences and Community Health, University of Milan, Milan, Italy. ${ }^{18}$ Interstitial Lung Disease Unit, Manchester University Hospital NHS FT, Wythenshawe Hospital, Manchester, UK. ${ }^{19}$ Dept of Clinical and Molecular Sciences, Università Politecnica delle Marche, Ancona, Italy.

Correspondence: Martina Bonifazi, Dept of Biomedical Sciences and Public Health, Università Politecnica delle Marche, Via Tronto 10/a, Ancona, Italy. E-mail: bonifazimartidgmail.com 
non-Raynaud's phenomenon clinical manifestation), cutaneous subset (limited/diffuse), serum autoantibodies, pulmonary hypertension (defined as a systolic pulmonary arterial pressure $\geqslant 40 \mathrm{mmHg}$ by echocardiography and/or a mean pulmonary artery pressure $\geqslant 25 \mathrm{mmHg}$ by right heart catheterisation), previous and/or concomitant treatments (corticosteroids and/or immunosuppressant drugs). Treatment status was sub-categorised as either "intention to treat" (treatment instituted within 3 months of presentation or continuation of pre-existing treatment) or "intention to observe" (no therapy at 3 months of follow-up).

PFT measurements (expressed as absolute value and percent predicted) were recorded if performed within 6 months of chest HRCT. They included forced vital capacity (FVC), forced expiratory volume in $1 \mathrm{~s}$ $\left(\mathrm{FEV}_{1}\right)$, and diffusing capacity of the lungs for carbon monoxide $\left(D_{\mathrm{LCO}}\right)$. The composite physiological index $(\mathrm{CPI})$ was calculated using the formula: $91.0-\left(0.65 \times \%\right.$ predicted $\left.D_{\mathrm{LCO}}\right)-(0.53 \times \%$ predicted $\mathrm{FVC})+$ $\left(0.34 \times \%\right.$ predicted $\left.\mathrm{FEV}_{1}\right)$.

The presence of either limited or extensive ILD involvement in SSc was defined according to the staging system proposed by $\mathrm{GoH}$ et al. [3], based on integration of HRTC evaluation and FVC estimation (supplementary material).

For the RBH cohort, all sequential lung function tests, routinely collected and available from baseline until last visit or death were also analysed. Trends were analysed as continuous change and as categorical change in separate models. FVC and $D_{\mathrm{LCO}}$ changes were computed as the percentage change relative to the absolute values at baseline. We did not record longitudinal lung function measurements in the Ancona cohort, as most patients did not undergo routine PFTs on follow up.

\section{HRCT analysis}

HRCT scans were evaluated independently by two experienced radiologists, with a third scorer adjudicating discordant scores. Scorers assessed PPFE features and extent [13], total ILD extent as well as freestanding bronchial abnormalities $[12,15,16]$. The extent of pleural surface involvement from PPFE in each lobe was evaluated on a four-point categorical scale. PPFE extent was further classified as limited $(\leqslant 2 / 18)$ or extensive $(>2 / 18)$. Further details of the scoring methodology are provided in the supplementary material.

\section{Statistical analysis}

Analyses were performed using SAS (version 9.4). Group comparisons were made using Student's t-test, Wilcoxon rank sum, Chi-squared statistics and Fisher's exact test, as appropriate. Interobserver variation for visual score between radiologists was assessed using weighted $\kappa$ statistics.

Mortality and disease progression were quantified from the date of the first available HRCT up to March 2018. Univariable and multivariable Cox proportional hazard analysis was undertaken to investigate determinants of mortality. Multivariable models always included terms for age, sex, cohort, PPFE, GOH et al. [3] staging system or CPI, FVC, $D_{\mathrm{LCO}}$, active treatment, pulmonary hypertension, body mass index (BMI) and smoking status. We considered the composite categorical decline (CCD) at 2 years, defined as either a decline in FVC of $\geqslant 10 \%$ or a decline in FVC of $5-9 \%$ in combination with a decline in $D_{\mathrm{LCO}}$ of $\geqslant 15 \%$. This was included as time-dependent covariate in multivariable survival analysis.

The relationship between PPFE and the annual rate of decline in absolute FVC values (measured as millilitres per year) from the date of HRCT to the end of follow up, with all available FVC values included, was analysed by using a random coefficient regression model (with random slopes and intercepts) that included sex and age as covariates.

\section{Results}

Of the initial 705 patients screened, 261 were excluded as the chest HRCT was not available for assessment. However, no differences in ILD severity were seen between patients included in the study and those excluded because of unavailable HRCT (supplementary material). Of the remaining 444 patients, 45 were excluded because of overlap with other connective tissue diseases and 40 due to the presence of predominant vasculopathy features on HRCT. A total of 359 patients were included in the present study, of which 228 from RBH and 131 from Ancona (figure 1).

Demographic and clinical characteristics of the combined cohort and by centre are summarised in table 1. The mean \pm SD age was $53.6 \pm 13.2$ years, and the majority of patients were females $(76.8 \%)$ and nonsmokers (58\%), without significant differences according to cohort. Disease duration was similar in the two cohorts (74.9 \pm 85.6 and $62.8 \pm 86.5$ months), the limited cutaneous subtype was more frequent than the diffuse in both cohorts, and no substantial difference was detected in anti-topoisomerase antibody frequency. Lung involvement was significantly worse in the $\mathrm{RBH}$ cohort, as documented by worse lung function 
FIGURE 1 Flow-chart of cohort selection. RBH: Royal Brompton Hospital Interstitial Lung Diseases Unit.

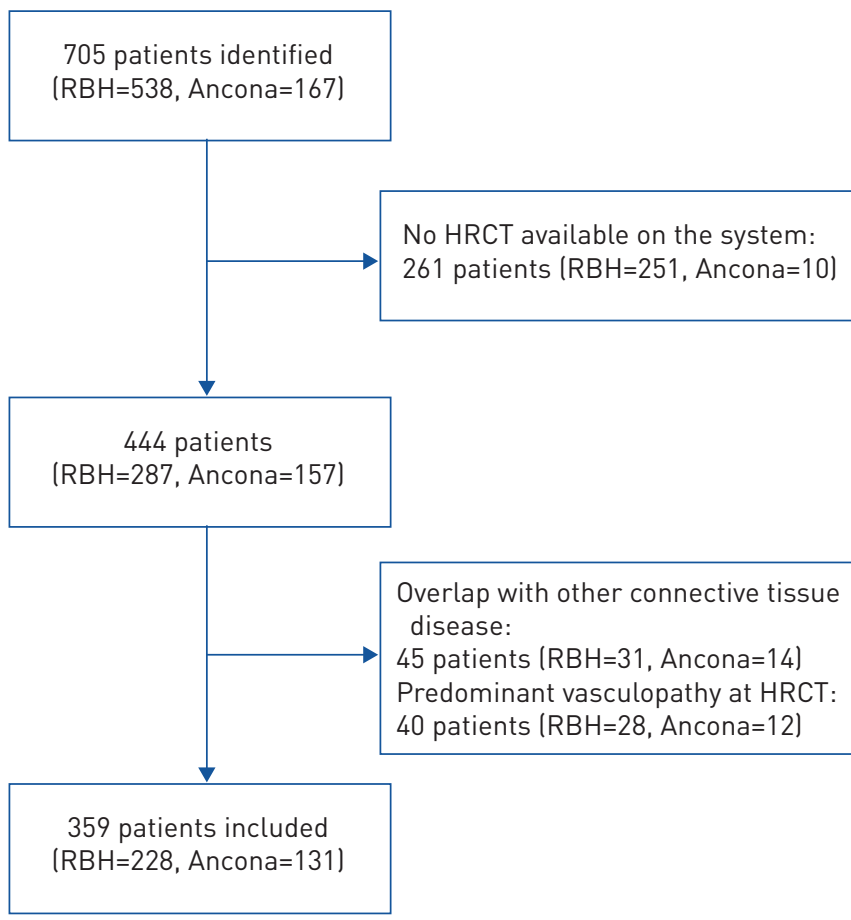

TABLE 1 Demographic, clinical and radiological characteristics of the whole cohort and by centre

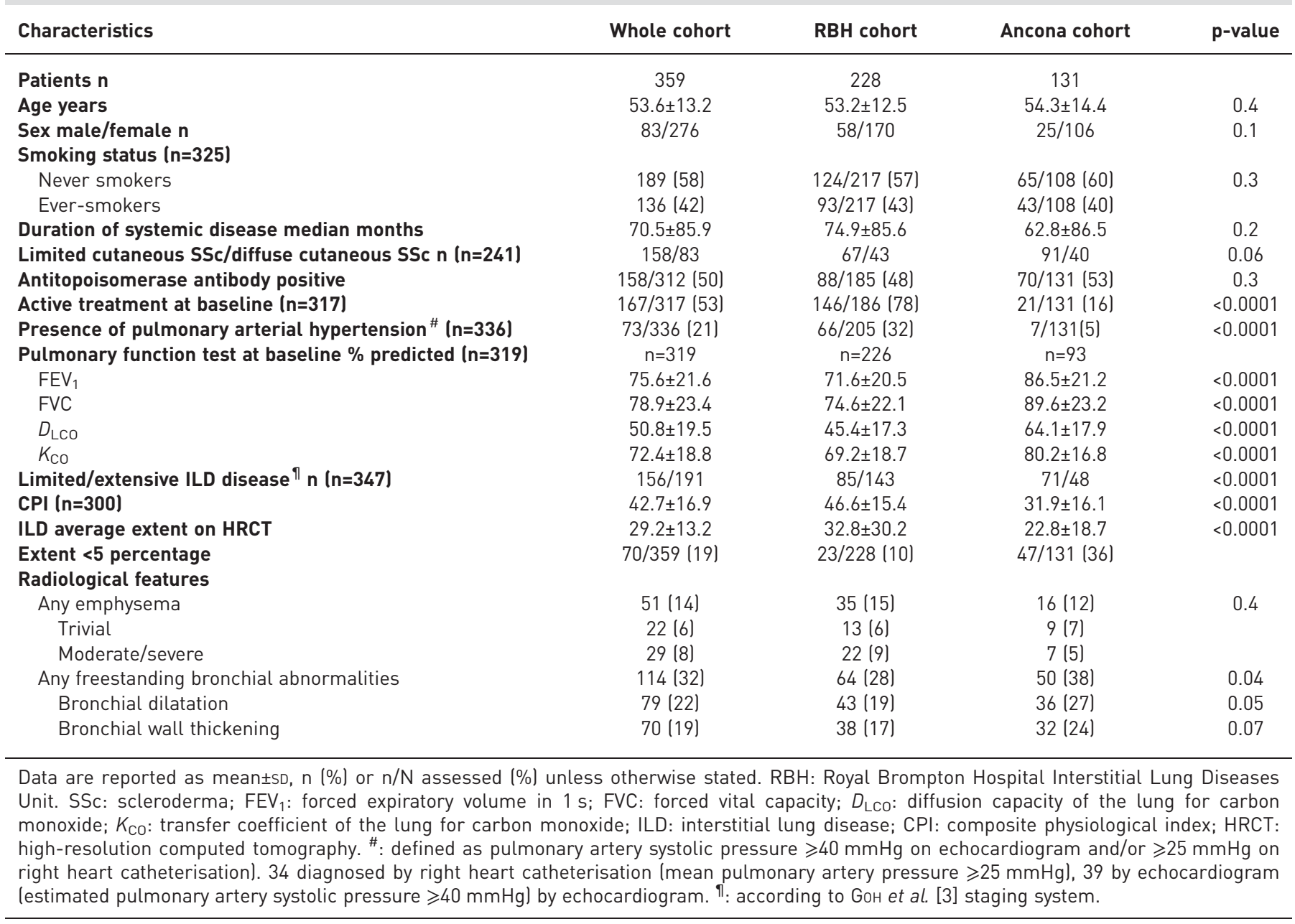


parameters, higher mean CPI, greater ILD extent on HRCT, more frequent extensive ILD stage [3], prevalence of pulmonary hypertension, and higher proportion of subjects on "intention to treat" (table 1). Median follow up time was 6.7 years in $\mathrm{RBH}$ cohort and 8.6 years in the Ancona cohort. With reference to radiological features other than PPFE, the prevalence of emphysema was approximately $15 \%$, with no substantial difference between subgroups. Freestanding bronchial abnormalities were overall present in up to one-third of the study population (32\%), with a higher proportion in the Ancona cohort (respectively $38 \%$ and $28 \%)$.

Prevalence of PPFE and associations with selected features

PPFE was detected in $65(18.1 \%)$ out of 359 SSc patients (PPFE+), of whom $41(11.4 \%)$ had extensive PPFE The prevalence of PPFE, overall and by extent (limited/extensive) was similar in the two cohorts (table 2). Examples of limited and extensive PPFE are provided in figure 2. Interobserver agreement for the presence of PPFE was good (weighted $\kappa$ statistics: 0.67). A comparison of demographic, clinical and radiological characteristics according to the presence of PPFE is shown in table 3. Age, sex, smoking status, cutaneous subtype, anti-topoisomerase antibody frequency, active treatment and presence of pulmonary hypertension did not differ between PPFE+ and PPFE- subgroups. PPFE was associated with a trend towards longer disease duration, a slightly lower mean ILD extent at HRCT, and more severe functional impairment (in FVC and $D_{\text {LCO }}$ predicted values), although these differences did not reach statistical significance.

Body mass index (BMI) was significantly lower in PPFE+ compared to PPFE- patients $(21.2 \pm 3.53$ and $25.3 \pm 4.91$, respectively). The prevalence of freestanding bronchial abnormalities was markedly higher in patients with PPFE compared to those without (61\% in PPFE+ group versus $25 \%$ in PPFE- group; $\mathrm{p}<0.0001)$. There was no statistical difference in the prevalence of emphysema according to PPFE presence (14\% in patients with and without PPFE).

\section{Prognostic impact of PPFE}

Data on survival were available for 344 patients (PPFE - subgroup=281, PPFE + subgroup=63) (figure 3). As no difference was observed in the prevalence of PPFE or in its severity according to centre, the analysis of the relationship between PPFE and survival was performed using the combined cohorts, although cohorts were adjusted for in the multivariable analysis. The presence of PPFE on HRCT was significantly associated with increased mortality on univariable analysis (HR: 1.56, 95\% CI 1.02-2.40; $\mathrm{p}=0.04$ ) (figure 1 and supplementary table S2). There were 28 deaths in PPFE+ subgroup (44\%) and 92 in PPFE- subgroup (32\%). Multivariable analyses are reported in table 4, providing a base model, adjusted for age, sex, cohort and GoH staging system, and a full model, including also active treatment, pulmonary hypertension, BMI and smoking status. The association, if anything, was stronger in both models (HR 1.79, 95\% CI 1.162.78; $\mathrm{p}=0.009$ in base model; HR $1.89,95 \%$ CI $1.10-3.25 ; \mathrm{p}=0.02$ in full model) (table 4 ). In order to assess whether the relationship with PPFE was influenced by short-term lung function changes, the full model also included the CCD variable, with no change in the association between PPFE and survival (table 4). When CPI was used in a separate multivariable model, the association between PPFE and survival remained significant (HR 1.60, 95\% CI 1.03-2.50; $\mathrm{p}=0.04$ ).

With regard to the severity of PPFE, we observed a trend towards a worse survival both in patients with limited and with extensive PPFE, compared with PPFE- patients; although, in view of the small numbers in each subgroup (table S2), this did not reach statistical significance on either univariable on multivariable analyses.

In the RBH cohort alone, we analysed the relationship between PPFE and the adjusted annual rate of decline in absolute FVC values. A trend towards higher decline in absolute FVC values (66 mL.year ${ }^{-1}$

TABLE 2 PPFE prevalence and extent in the whole cohort and by centre

\begin{tabular}{lcccc} 
Characteristics & Whole cohort & RBH cohort & Ancona cohort & p-value \\
\hline Patients n & 359 & 228 & 131 & \\
PPFE & $65(18.1)$ & $42(18.4)$ & $23(17.5)$ & 0.8 \\
Limited & $24(6.7)$ & $16(7.0)$ & $8(6.1)$ & $15(11.4)$ \\
Extensive & $41(11.4)$ & $26(11.4)$ &
\end{tabular}

Data are presented as $\mathrm{n}(\%)$, unless otherwise stated. RBH: Royal Brompton Hospital Interstitial Lung Diseases Unit. PPFE: pleuroparenchymal fibroelastosis. 


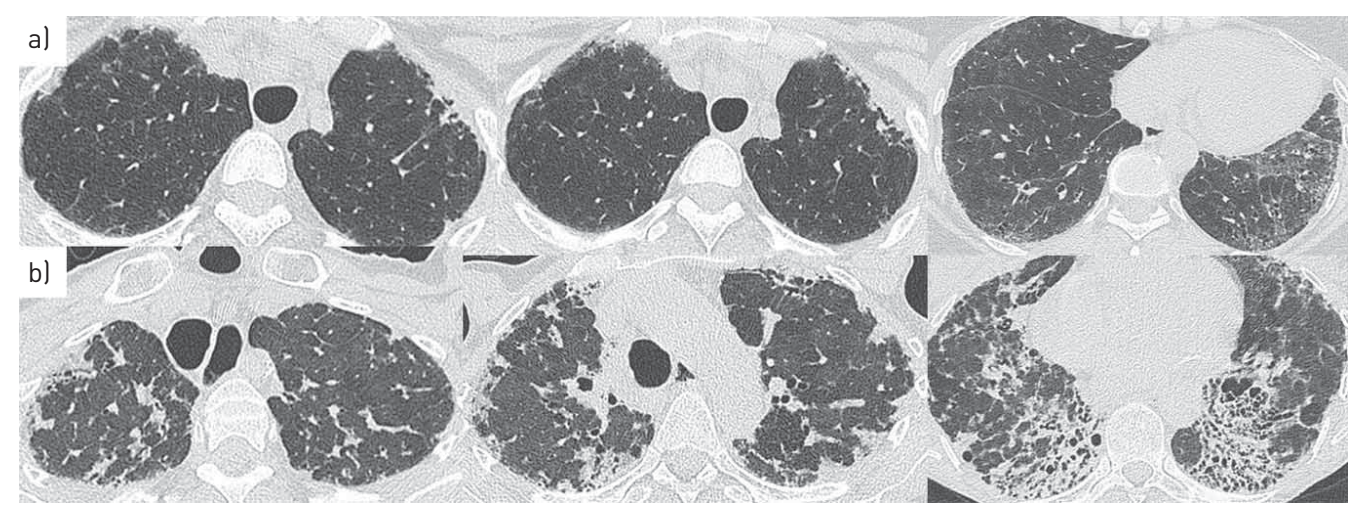

FIGURE 2 a and b) Axial computed tomography images showing limited (a) and extensive (b) features of pleuraparenchymal fibroleastosis (PPFE). a) Limited pleural and parenchymal aggregations of fibrous tissue in the upper lobes, and separate interstitial abnormalities in the lower lobes. b) Extensive subpleural, parenchymal and airways-centred PPFE features in the upper and lower lobes. A fibrotic nonspecific interstitial pneumonia (NSIP) pattern can be also observed in the lower lobes.

versus $44 \mathrm{~mL} \cdot \mathrm{year}^{-1} ; \mathrm{p}=0.08$ ) was detected, although only of borderline significance. This did not change after adjusting for disease extent or "intention to treat" (data not shown).

\section{Discussion}

The present study provides a detailed assessment of PPFE prevalence, associations and clinical impact in a large cohort of SSc patients, derived from two referral centres in two different countries. PPFE was present in approximately $18 \%$ of patients and was an independent predictor of worse prognosis.

\section{TABLE 3 Demographic, clinical and radiological characteristics according to PPFE presence}

\begin{tabular}{|c|c|c|c|}
\hline Characteristics & $\begin{array}{l}\text { Patients with PPFE } \\
\qquad(n=65)\end{array}$ & $\begin{array}{l}\text { Patients without PPFE } \\
\text { (n=294) }\end{array}$ & p-value \\
\hline Age years & $54.3 \pm 11.5$ & $53.4 \pm 13.5$ & 0.6 \\
\hline Sex male/female $n$ & $10 / 55$ & $73 / 221$ & 0.1 \\
\hline Smoking status ( $n=315$ ) & $n=56$ & $n=259$ & \\
\hline Never smokers & $33(59)$ & 146 (49) & 0.6 \\
\hline Ever-smokers & $23(41)$ & $113(43)$ & \\
\hline Body mass index $(n=328)$ & $21.2 \pm 3.52$ & $25.3 \pm 4.91$ & $<0.0001$ \\
\hline Duration of systemic disease months & $87.3 \pm 89.2$ & $66.8 \pm 89.2$ & 0.08 \\
\hline Limited cutaneous SSc $(n=241)$ & $25 / 40(62)$ & $133 / 201(66)$ & 0.6 \\
\hline Antitopoisomerase antibody positive $(n=316)$ & $28 / 57(50)$ & $130 / 259(50)$ & 0.3 \\
\hline Intention to treat $(n=317)$ & $30 / 59(50)$ & $137 / 258(53)$ & 0.7 \\
\hline $\begin{array}{l}\text { Presence of pulmonary arterial } \\
\text { hypertension }{ }^{\#}(n=336)\end{array}$ & $15 / 61(25)$ & $58 / 275(21)$ & 0.6 \\
\hline $\begin{array}{l}\text { Pulmonary function test at baseline } \\
\% \text { predicted }(n=319)\end{array}$ & $n=59$ & $n=260$ & \\
\hline FVC & $74.5 \pm 24.6$ & $80.0 \pm 23.1$ & 0.1 \\
\hline$D_{\mathrm{LCO}}$ & $47.6 \pm 17.3$ & $51.5 \pm 19.8$ & 0.1 \\
\hline$K_{\mathrm{CO}}$ & $72.9 \pm 19.9$ & $72.3 \pm 18.6$ & 0.8 \\
\hline ILD average extent on HRCT & $26.9 \pm 21.3$ & $29.7 \pm 22.7$ & 0.3 \\
\hline \multicolumn{4}{|l|}{ Radiological features } \\
\hline Any emphysema & $9(14)$ & $42(14)$ & 0.9 \\
\hline Any freestanding bronchial abnormalities & $40(61)$ & $74(25)$ & $<0.0001$ \\
\hline Bronchial dilatation & $31(48)$ & $48(16)$ & $<0.0001$ \\
\hline Bronchial wall thickening & $27(42)$ & $43(14)$ & $<0.0001$ \\
\hline
\end{tabular}

Data are reported as mean $\pm \mathrm{SD}, \mathrm{n}(\%)$ or $\mathrm{n} / \mathrm{N}$ assessed $(\%)$, unless otherwise stated. PPFE: pleuroparenchymal fibroelastosis; SSc: scleroderma; FVC: forced vital capacity; $D_{\text {Lco: }}$ diffusion capacity of the lung for carbon monoxide; $K_{\mathrm{co}}$ : transfer coefficient of the lung for carbon monoxide; ILD: interstitial lung disease; HRCT: high-resolution computed tomography. \#: defined as pulmonary artery systolic pressure $\geqslant 40 \mathrm{mmHg}$ on echocardiogram and/or $\geqslant 25 \mathrm{mmHg}$ on right heart catheterisation). 


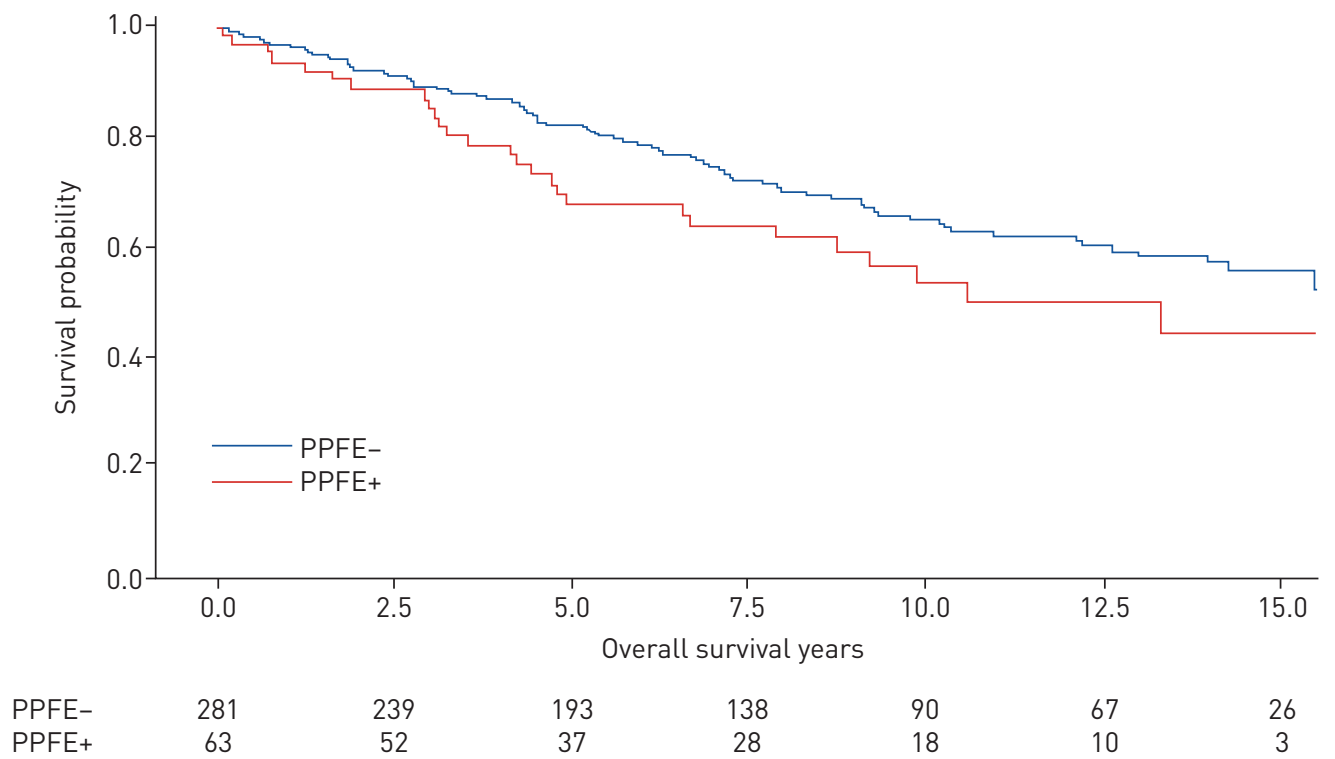

FIGURE 3 Survival comparison between patients with and without pleuraparenchymal fibroleastosis (PPFE) in the whole cohort ( $n=344)$.

Interestingly, the prevalence of PPFE was essentially the same in the two populations, despite a significant difference in ILD severity. The RBH cohort was derived from a tertiary ILD referral centre and thus characterised by more severe ILD compared with the Ancona cohort, which was retrieved from a rheumatology referral centre for SSc, less selected in terms of pulmonary involvement. This suggests that the genesis and progression of PPFE in SSc is not necessarily linked to the progression of the background

TABLE 4 Mortality in SSc patients according to selected covariates, expressed as hazard ratios (HR) with 95\% confidence intervals

\begin{tabular}{|c|c|c|c|c|c|}
\hline \multirow[b]{2}{*}{ Characteristic } & \multicolumn{3}{|c|}{ Base model ${ }^{\#}$} & \multicolumn{2}{|c|}{ Full model ${ }^{\pi}$} \\
\hline & $\mathbf{n}$ & HR & $95 \% \mathrm{Cl}$ & HR & $95 \% \mathrm{Cl}$ \\
\hline PPFE (yes) & 332 & 1.79 & $1.16-2.78$ & 1.89 & $1.10-3.25$ \\
\hline Age (years) & 332 & 1.04 & $1.02-1.06$ & 1.05 & $1.03-1.07$ \\
\hline Sex (female) & 332 & 1.57 & $1.05-2.33$ & 1.71 & $1.05-2.77$ \\
\hline Cohort (Italian) & 332 & 0.43 & $0.26-0.69$ & 0.57 & $0.21-0.53$ \\
\hline FVC $\%$ pred (per $1 \%$ increase) & 306 & 0.97 & $0.96-0.98$ & 1.00 & $0.98-1.01$ \\
\hline$D_{\text {Lco }} \%$ pred (per $1 \%$ increase) & 304 & 0.94 & $0.93-0.96$ & 0.95 & $0.92-0.97$ \\
\hline ILD extent at HRCT (per $1 \%$ increase) & 344 & 1.02 & $1.01-1.03$ & 1.01 & $0.99-1.03$ \\
\hline GoH et al. staging system (extensive) & 332 & 2.15 & $1.42-3.26$ & 2.38 & $1.29-4.40$ \\
\hline CPI (per unit increase) & 288 & 1.05 & $1.03-1.06$ & 1.05 & $1.03-1.08$ \\
\hline CCD at 2 years $^{+}$(yes) & 332 & 4.14 & $2.85-6.00$ & 5.95 & $3.56-8.78$ \\
\hline Active treatment (yes) & 296 & 1.24 & $0.69-2.21$ & 1.71 & $0.83-3.49$ \\
\hline Pulmonary hypertension $\S$ (yes) & 309 & 2.78 & $1.85-4.24$ & 3.07 & $1.93-4.89$ \\
\hline Smoking status (ever) & 284 & 1.09 & $0.72-1.63$ & 0.99 & $0.62-1.61$ \\
\hline BMI (per $\mathrm{kg} \cdot \mathrm{m}^{-2}$ ) & 306 & 0.95 & $0.90-0.99$ & 0.96 & $0.92-1.01$ \\
\hline
\end{tabular}

PPFE: pleuroparenchymal fibroelastosis; FVC: forced vital capacity; $D_{\mathrm{LCo}}$ : diffusion capacity of the lung for carbon monoxide; ILD: interstitial lung disease; HRCT: high-resolution computed tomography; PH: arterial pulmonary hypertension (defined as PASP $\geqslant 40 \mathrm{mmHg}$ on echocardiogram and/or $\geqslant 25 \mathrm{mmHg}$ on right heart catheterisation); SSc: scleroderma. ${ }^{\#}$ : hazard ratios adjusted for age, sex, cohort, and GoH staging, except for ILD extent at HRCT and composite physiological index (CPI). ": hazard ratios adjusted for all the characteristics listed in the table, except for ILD extent at HRCT, FVC \%, $D_{\mathrm{LCO}} \%$, and CPI ( $\left.\mathrm{n}=227\right)$. For ILD extent at HRCT and, in a separate model CPI, hazard ratios adjusted for all the characteristics listed in the table except for $\mathrm{GOH}$ staging, $D_{\mathrm{LCO}} \%$ and FVC \% (n=216). ${ }^{+}$: time-dependent covariate. ${ }^{\S}$ : defined as pulmonary artery systolic pressure $\geqslant 40 \mathrm{mmHg}$ on echocardiogram and/or $\geqslant 25 \mathrm{mmHg}$ on right heart catheterisation). 
ILD pattern and may have alternative explanations. Interestingly, we observed a strong association between PPFE and freestanding bronchial abnormalities, although this link remains to be explained.

These results are in line with a previous Japanese study evaluating prevalence and prognosis of radiological PPFE lesions in a cohort of patients with connective tissue disease (CTD)-ILDs ( $\mathrm{n}=113$ ), including 14 subjects with SSc. The overall prevalence of PPFE in the whole CTD population was 19\%, with the highest peak in the SSc subgroup (43\%); survival analysis in a multivariable model showed that PPFE presence was a significant risk factor for mortality due to respiratory causes [9]. We also found that the presence of PPFE features is independently associated with an excess mortality risk by $77 \%$ on multivariable analysis that included age, GoH et al. [3] staging system (or CPI in separate models), BMI, progressive functional decline (CCD at 2 years), intention to treat and pulmonary hypertension. Potential determinants of a worse survival may include direct mechanisms, such as the marked functional restriction in these patients and/or more rapid lung function decline, as well as indirect/systemic effects, such as a reduced BMI and/or higher susceptibility to infections. A more rapid rate of lung function decline is a further pathological mechanism that might be linked to a higher mortality, as we observed a trend bordering on statistical significance towards a greater rate of decline in FVC in patients with PPFE. However, as longitudinal lung function was only available for the RBH cohort, further studies are needed to assess the link between PPFE and lung function decline in different populations. We find a strong association with low BMI in PPFE+ subjects, with BMI predictive of worse survival on univariable analysis in our cohort. Although the association was no longer significant on multivariable analysis, a low BMI may well be contributing to the poor survival in this cohort. A low or decreasing BMI is known to be an independent predictor of increased mortality in patients with IPF [17-20].

With reference to the higher susceptibility to infections in these patients, it is worth underlining the strong association between PPFE and freestanding bronchiectasis, found in both our cohorts. The higher prevalence of freestanding bronchial abnormalities in subjects with PPFE (33.7\% versus $1.1 \%$; $<<0.0001)$ is consistent with findings in a large IPF cohort $(n=274)[10]$. The strong link between PPFE and freestanding bronchiectasis supports the hypothesis that PPFE might represent an aberrant fibrosing immune response to repeated infections and inhaled antigens. In the paper by ReDDY et al. [13], over half of PPFE patients reported recurrent infections, including allergic bronchopulmonary aspergillosis and aspergilloma, and PICIUCCHI et al. [21] documented a PPFE case of a patient who tested positive for Aspergillus spp. precipitins. Moreover, no significant association was found between PPFE and smoking history, confirming previous observations [12].

We did not observe increased mortality in extensive PPFE, compared with limited PPFE, despite the fact that the extensive PPFE has been linked to higher mortality in IPF and hypersensitivity pneumonitis $[10,12]$. This is possibly due to the low number of observations, or to a different interaction operating in SSc-ILD patients.

The study had limitations. Although the computed tomographic appearance of PPFE has been defined [13], tissue corroboration was lacking in our cohorts. However, as the histological distinction between a UIP and NSIP pattern does not change management in SSc-ILD [22, 23], tissue biopsy is no longer performed in these patients. Thus, PPFE can be identified in SSc patients only by means of HRCT and, therefore, our observations are applicable to routine clinical practice with PPFE identified with observed good inter-observer agreement. Selection bias must be acknowledged, as patients were evaluated at two national tertiary referral centres and were, therefore, likely to have more severe pulmonary involvement than in unselected cohorts. Furthermore, there were differences in patient characteristics between the two centres. However, the presence of PPFE was not related to ILD severity and, importantly, the linkage between PPFE and mortality was independent of ILD severity, the distinction between intention to treat and intention to observe, and the referral centre. Given these observations and the fact that the full spectrum of ILD severity was well represented in the study cohort, we believe that our findings are likely to be generalisable.

A further potential bias is related to the exclusion of subjects with predominant features suggestive of vasculopathy at HRCT. In the absence of standardised radiological criteria, we adopted the features used to describe SSc-associated pulmonary veno-occlusive disease, including the presence of both centrilobular ground-glass opacities and non-subpleural interlobular septal thickening [24, 25]. As indirect confirmation of the reliability of this definition in identifying vascular rather than interstitial changes, a reduction in $D_{\text {LCO }}$ with preserved lung volumes was observed in this excluded subgroup. Lastly, due to the retrospective nature of this study, information on serological status was missing for many patients, and standardised data on the frequency of respiratory infections and other clinical features was not available.

In conclusion, the increasing awareness of PPFE among specialists over recent years has led to an increase in its identification in both idiopathic and secondary contexts, suggesting that it is not as rare as previously thought. In the current study, we describe PPFE prevalence, extent and clinical impact in the 
largest cohort of SSc subjects published so far. Our results indicate that its presence should be carefully considered, due to its significant prognostic implications. Owing to the absence of effective tailored treatments, the identification of PPFE does not currently alter management of this subgroup of SSc patients, but greater awareness, surveillance and careful prevention of infections is recommended. Further studies are needed to better define the pathogenesis and optimal management of PPFE.

Acknowledgement: We thank David Hansell for his invaluable contribution to the design of this study.

Conflict of interest: M. Bonifazi reports personal fees from Boehringer Ingelheim and Roche, outside the submitted work. N. Sverzellati reports personal fees from Boehringer Ingelheim and Roche, outside the submitted work. E. Negri has nothing to disclose. J. Jacob reports personal fees from Roche and Boehringer Ingelheim, grants from GlaxoSmithKline, outside the submitted work. R. Egashira reports personal fees and non-financial support from Shionogi, Boehringer Ingelheim Japan, Bayer Yakuhin, Eisai, Daiichi Sankyo and AstraZeneca, non-financial support from Kyorin, outside the submitted work. J. Moser has nothing to disclose. S. Piciucchi has nothing to disclose. F. Mei has nothing to disclose. A. De Lauretis has nothing to disclose. D. Visca has nothing to disclose. N. Goh has nothing to disclose. M. Bonini has nothing to disclose. L. Cirilli has nothing to disclose. C. La Vecchia has nothing to disclose. F. Chua has no conflict of interest to declare V. Kouranos has nothing to disclose. G. Margaritopoulos has nothing to disclose. M. Kokosi has nothing to disclose. T.M. Maher has, via his institution, received industry-academic funding from GlaxoSmithKline R\&D and UCB and has received consultancy or speakers fees from Apellis, Astra Zeneca, Bayer, Blade Therapeutics, Boehringer Ingelheim, Bristol-Myers Squibb, Galapagos, GlaxoSmithKline R\&D, Indalo, Novartis, Pliant, ProMetic, Respivnat, Roche, Samumed and UCB. S. Gasparini has nothing to disclose. A. Gabrielli has nothing to disclose. A.U. Wells reports personal fees from Boehringer Ingelheim, Roche and Bayer, outside the submitted work. E.A. Renzoni reports personal fees from Boehringer Ingelheim and Roche, outside the submitted work.

Support statement: This work was supported in part by Versus Arthritis and by Scleroderma and Raynaud's UK. Joseph Jacob was funded by Wellcome Trust Clinical Research Career Development Fellowship 209553/Z/17/Z.

\section{References}

Desbois AC, Cacoub P. Systemic sclerosis: An update in 2016. Autoimmun Rev 2016; 15: 417-426.

2 Steen VD, Medsger TA. Changes in causes of death in systemic sclerosis, 1972-2002. Ann Rheum Dis 2007; 66: 940-944.

3 Goh NS, Desai SR, Veeraraghavan S, et al. Interstitial lung disease in systemic sclerosis: a simple staging system. Am J Respir Crit Care Med 2008; 177: 1248-1254.

4 Goh NS, Hoyles RK, Denton CP, et al. Short-term pulmonary function trends are predictive of mortality in interstitial lung disease associated with systemic sclerosis. Arthritis Rheumatol 2017; 69: 1670-1678.

5 Denton CP, Khanna D. Systemic sclerosis. Lancet 2017; 390: 1685-1699.

6 Bonifazi M, Montero MA, Renzoni EA. Idiopathic pleuroparenchymal fibroelastosis. Curr Pulmonol Rep 2017; 6: 9-15.

7 Travis WD, Costabel U, Hansell DM, et al. An official American Thoracic Society/European Respiratory Society statement: Update of the international multidisciplinary classification of the idiopathic interstitial pneumonias. Am J Respir Crit Care Med 2013; 188: 733-748.

8 Mariani F, Gatti B, Rocca A, et al. Pleuroparenchymal fibroelastosis: the prevalence of secondary forms in hematopoietic stem cell and lung transplantation recipients. Diagn Interv Radiol 2016; 22: 400-406.

9 Enomoto Y, Nakamura Y, Colby TV, et al. Radiologic pleuroparenchymal fibroelastosis-like lesion in connective tissue disease-related interstitial lung disease. PLoS One 2017; 12: e0180283.

10 De Lauretis A, Basra H, Hakim W. Pleuroparenchymal Fibroelastosis (PPFE) Predicts Survival in Idiopathic Pulmonary Fibrosis (IPF). Am J Respir Crit Care Med 2016; 193: A1142.

11 Oda $\mathrm{T}$, Ogura $\mathrm{T}$, Kitamura $\mathrm{H}$, et al. Distinct characteristics of pleuroparenchymal fibroelastosis with usual interstitial pneumonia compared with idiopathic pulmonary fibrosis. Chest 2014; 146: 1248-1255.

12 Jacob J, Odink A, Brun AL, et al. Functional associations of pleuroparenchymal fibroelastosis and emphysema with hypersensitivity pneumonitis. Respir Med 2018; 138: 95-101.

13 Reddy TL, Tominaga M, Hansell DM, et al. Pleuroparenchymal fibroelastosis: a spectrum of histopathological and imaging phenotypes. Eur Respir J 2012; 40: 377-385.

14 van den Hoogen F, Khanna D, Fransen J, et al. 2013 classification criteria for systemic sclerosis: an American College of Rheumatology/European League against Rheumatism collaborative initiative. Arthritis Rheum 2013; 65: 2737-2747.

15 Cowman SA, Jacob J, Hansell DM, et al. Whole-blood gene expression in pulmonary nontuberculous mycobacterial infection. Am J Respir Cell Mol Biol 2018; 58: 510-518.

16 de Jong PA, Ottink MD, Robben SG, et al. Pulmonary disease assessment in cystic fibrosis: comparison of CT scoring systems and value of bronchial and arterial dimension measurements. Radiology 2004; 231: 434-439.

17 Kim JH, Lee JH, Ryu YJ, et al. Clinical predictors of survival in idiopathic pulmonary fibrosis. Tuberc Respir Dis (Seoul) 2012; 73: 162-168.

18 Kulkarni T, Yuan K, Tran-Nguyen TK, et al. Decrements of body mass index are associated with poor outcomes of idiopathic pulmonary fibrosis patients. PLoS One 2019; 14: e0221905.

19 Kishaba T, Nagano H, Nei Y. Body mass index-percent forced vital capacity-respiratory hospitalization: new staging for idiopathic pulmonary fibrosis patients. J Thorac Dis 2016; 8: 3596-3604.

20 Jouneau S, Lederlin M, Vernhet L, et al. Malnutrition in idiopathic pulmonary fibrosis: the great forgotten comorbidity!. Eur Respir J 2019; 53: 1900418.

21 Piciucchi S, Tomassetti S, Casoni G, et al. High resolution CT and histological findings in idiopathic pleuroparenchymal fibroelastosis: features and differential diagnosis. Respir Res 2011; 12: 111.

22 Bouros D, Wells AU, Nicholson AG, et al. Histopathologic subsets of fibrosing alveolitis in patients with systemic sclerosis and their relationship to outcome. Am J Respir Crit Care Med 2002; 165: 1581-1586. 
23 Wells AU, Denton CP. Interstitial lung disease in connective tissue disease--mechanisms and management. Nat Rev Rheumatol 2014; 10: 728-739.

24 Duarte AC, Cordeiro A, Loureiro MJ, et al. Pulmonary veno-occlusive disease: a probably underdiagnosed cause of pulmonary hypertension in systemic sclerosis. Clin Rheumatol 2020; 39: 1687-1691.

25 Connolly MJ, Abdullah S, Ridout DA, et al. Prognostic significance of computed tomography criteria for pulmonary veno-occlusive disease in systemic sclerosis-pulmonary arterial hypertension. Rheumatology (Oxford) 2017; 56: 2197-2203. 